\title{
Teoría de conjuntos
}

Francisco Vera Fernández de Córdoba

Rev. Acad. Colomb. Cienc. Ex. Fis. Nat. 1942, 5 (18): 230-240.

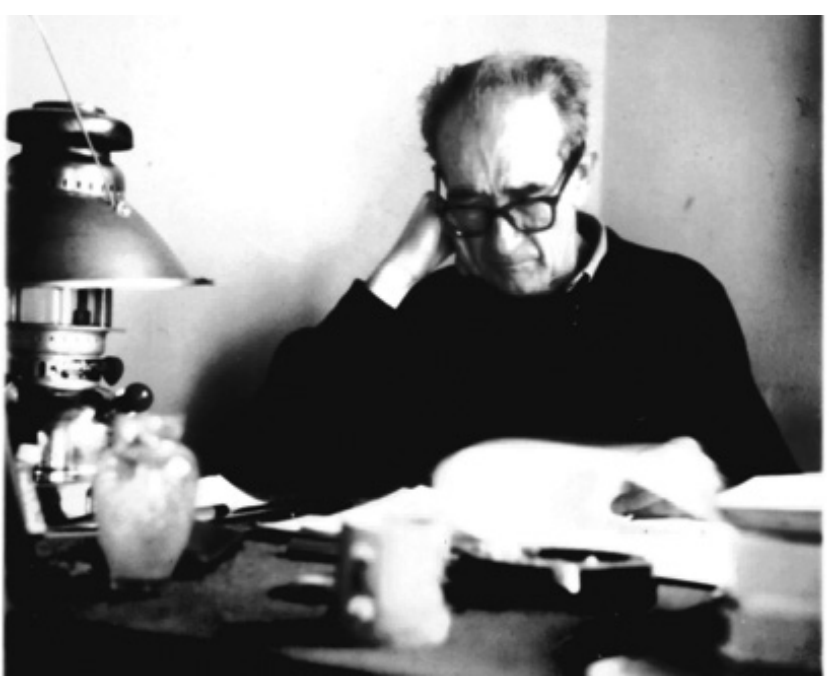

Francisco Vera Fernández de Córdoba (1888-1967)

Vera, fue un matemático español, prolífico escritor, reconocido especialmente por sus trabajos en historia de la ciencia y de la matemática. Exilado de la guerra civil española, llegó a Colombia por invitación del entonces presidente Eduardo Santos en abril de 1941. Fue profesor en la Escuela Normal Superior y en la Universidad Nacional, donde, entre 1941 y 1942, tuvo a su cargo cursos de aritmética analítica (esencialmente, teoría elemental de los números) en la Facultad de Ingeniería y posiblemente también de cálculo diferencial (Matemáticas II) en la Facultad de Química. Con el apoyo de la Sociedad Colombiana de Ingenieros llevó a cabo cursillos y conferencias de divulgación de las matemáticas, en la sede de la Sociedad, en la UN y en el Teatro Colón. Con sus cursos y conferencias abrió el camino para que nuestra comunidad académica y cultural se diera cuenta del grave atraso en que nos encontrábamos de lo cual dejó testimonio en algunas de sus obras.

\section{Referencias}

Albis V., Sánchez C.H. 2009 La introducción de la teoría de conjuntos y la matemática moderna en Colombia. Primera parte: El aporte de los extranjeros. Mathesis III. 42: 265-293

Cobos Bueno J.M, Vaquero Martínez J.M. 1999. "Matemáticas y exilio: la primera etapa americana de Francisco Vera" Llull 1999, 2245: 569-588.

Vera F. 1948. Introducción a la Teoría de Conjuntos. Buenos Aires: Editora y Distribuidora del Plata.
El artículo de la Revista de la Academia es la publicación de los dos primeros capítulos de Vera en su libro Introducción a la Teoría de Conjuntos, el cual comienza con una 'Advertencia al lector' donde afirma:

Esta obrita es la reconstrucción aproximada del curso que sobre teoría de conjuntos dicté durante los meses de septiembre y octubre de 1942 en Bogotá por honroso encargo de la Sociedad Colombiana de Ingenieros, la que realizaba el enorme esfuerzo de organizar conferencias de matemática pura puesto que la que se explicaba en la Universidad Nacional tenía carácter más concreto que abstracto, ya que no existía en Colombia la Facultad de Ciencias creada recientemente.

En seis capítulos que constituyen el libro, Vera hace una presentación histórico-filosófica de la teoría de conjuntos, deteniéndose en los puntos más relevantes, como son la existencia de diferentes tipos de infinito para mostrar que los racionales y los naturales tienen la misma cardinalidad, pero que los reales tienen una cardinalidad diferente a la de los naturales. No se detiene en la parte operativa de la teoría, ni ofrece ejercicios. Aunque contiene algunos errores en sus demostraciones, el artículo fue seleccionado por ser el primero realizado en Colombia sobre la teoría de conjuntos.

Vera también publicó en la Revista de la Academia el artículo El "Tertium non datur" en la matemática actual, (4 (14), 1941) sobre las escuelas filosóficas de la matemática que surgieron a comienzos del siglo XX como propuestas de solución de las paradojas de la teoría de conjuntos de Cantor. Se trata, entonces, de dos artículos pioneros en Colombia sobre temas de matemática y lógica, de ahí su enorme importancia. Sobre el paso de Vera por Colombia, Cobos y Vaquero, sus biógrafos, afirman: "Nos encontramos a un Vera que explica lo que de sobra era ya conocido en Europa pero que no había llegado aún a algunos países de Latinoamérica”, Latinoamérica.

Clara Helena Sánchez, Ph.D.

Miembro Correspondiente 


\section{TEORIA DE CONJUNTOS}

PROF. FRANCISCO VERA

I

NOCIONES FUNDAMENTALES

1. Consunto.-Corresponde a Cantor (Georg, 18451918, alemán) la gloria de haber creado una teoría sin antecedentes históricos y de tal trascendencia, que con razón ha dicho Denjoy (Arnaud, 1884. ...., francés) que es la mayor revolución que ha conocido la Matemática después de la que produjo el Cálculo infinitesimal.

Parece natural que iniciemos este cursillo estableciendo el concepto de conjunto, cuestión no exenta de dificultades. Cantor lo definió diciendo que es "una conexión determinada de diversos objetos de nuestra intuición o de nuestra mente, llamados ele. mentos del conjunto, en una totalidad", definición que contiene una palabra peligrosa: totalidad, porque es inconstituíble excepto cuando nombramos los elementos que se consideran en la totalidad, en cuyo caso sólo tiene el valor de una abreviatura, de modo que si en una habitación están Pedro, Juan, Antonio y Enrique, por ejemplo, y nadie más, podemos hablar de la totalidad o de todas las personas que hay en la habitación; pero ¿cómo establecer mediante la observación directa que todos los cuerpos se dilatan por la acción del calor? Incluso en la hipótesis de que en un cierto momento hubieran sido observados todos los cuerpos del mundo desde el punto de vista de su dilatabilidad no podríamos afirmar sin imprudencia que los cuerpos observados eran todos los cuerpos.

Claro es que tampoco podemos establecer por observación directa algunos conceptos físicos - molécula, átomo, protón, etc.-y, de acuerdo con Mach (Ernst, 1838-1916, austriaco), tendríamos que borrar del diccionario científico los enunciados en que intervienen tales conceptos, so pena de invadir el terreno de la Metafísica; pero si reflexionamos un poco comprenderemos que no hay en ello ningún inconveniente porque la Física da indicaciones sobre el empleo de las palabras inconstituíbles, tales qque, partiendo de enunciados en que figuran, se llega a enunciados en que no figuran, pero cuya certeza se puede comprobar experimentalmente.

No ocurre lo propio con la palabra todos, referida a los elementos de un conjunto, porque las leyes lógicas nos dicen que lo que es válido para todos lo es también para cada objeto particular, y, sin embargo, más adelante demostraremos la existencia de conjuntos que no cumplen esta ley, lo que nos obliga a fijar la significación de la palabra conjunto para evitar equívocos.

Admitiendo sin discusión que sabemos distinguir entre una cosa y varias cosas y que al prescindir de la naturaleza de cada cosa y de la posición de unas respecto de otras, si son varias, nacen en nuestro espíritu las ideas de unidad y pluralidad, asignaremos a la palabra coniunto el valor de pluralidad definida o determinada, y diremos que un conjunto está definido o determinado cuando cualesquiera que sean los elementos que consideremos, no exista para cada uno de ellos más que el dilema de pertenecer o no pertenecer al conjunto y para cada par de elementos no exista más que el dilema de estar formado o no estar formado por elementos distintos.

Aceptadas estas condiciones, consideraremos provisionalmente la definición cantoriana como un objeto matemático que podemos manejar sin peligro, y no sólo será ya legítimo hablar del "conjunto de personas que están en una habitación", o del "conjunto de átomos de hidrógeno y oxígeno que hay en un centímetro cúbico de agua", o del "conjunto de los pecados capitales", por ejemplo, sino también, abusando del lenguaje en aras de la comodidad, llamar conjunto a un sólo elemento: conjunto unidad, pero sin confundirlo con el elemento, e incluso concebir que no tenga ninguno: conjunto mulo o vacío.

Designaremos los conjuntos por letras mayúsculas aisladas y sus elementos por mayúsculas cursivas. Así, para expresar que el conjunto M está formado por los elementos $A, B, C, D$, escribiremos

$$
\mathrm{M}=A, B, C, D .
$$

Diremos que un conjunto $\mathrm{M}$ es parte (1) de otro $\mathrm{N}$ si todos los elementos de M pertenecen a $\mathrm{N} y$ hay en $\mathrm{N}$ elementos que no pertenecen a M. La parte de un conjunto se llama también subconjunto o conjunto parcial. Indicaremos la relación entre un conjunto $\mathrm{N}$ y una parte de él M escribiendo $\mathrm{M}<\mathrm{N}$.

2. Correspondencia.-Otro concepto del que hemos de hacer uso frecuente es el de correspondencia: convenio o ley tal que fijado un elemento de un conjunto dado, queden fijados uno o varios elementos de otro conjunto también dado, diciéndose correspondencia unívoca en aquel caso y plurívoca en éste. De los elementos de uno de los conjuntos diremos que son correspondientes u homólogos de los del otro, y si los elementos se corresponden uno a uno, es decir: si cada elemento de un conjunto tiene un homólogo y sólo uno en el otro, y recíprocamente, la correspondencia se llama biunívoca.

(1) Cantor lo llama parte integrante: Bestandtheil. 
3. Orden.-Dado un elemento $A$ y otro elemento distinto $B$, diremos que uno de ellos, $A$, por ejemplo, precede al otro $B$ o que éste sigue a aquél, o bien que $A$ es anterior a $B$ o $B$ es posterior a $A$, y escribiremos $A<B$ o $B>A$. En esta definición los verbos preceder y seguir y los adverbios anterior y posterior sólo tienen el valor de símbolos.

Si un elemento $A$ de un conjunto es anterior a otro $B$, que no tiene ninguno anterior distinto de $A$, se dice que $\left\{\begin{array}{l}A \\ B\end{array}\right\}$ es el inmediatamente $\left\{\begin{array}{lll}\text { anterior a } & B \\ \text { posterior a } & A\end{array}\right\}$ y el elemento $B$ recibe el nombre de siguiente o consecutivo a A.

Diremos que un conjunto está ordcnado si podemos fijar un criterio cualquiera que permita decir sin ambigüedad si un elemento $A$ del conjunto es anterior a otro $B \quad y$, además, que si es

$$
A<B \quad \text { y } B<C \text {, sea } A<C \text {. }
$$

Ordenado un conjunto, si contiene un elemento al cual no precede ninguno y otro elemento al cual no sigue ninguno, diremos que aquél es el primero y éste el último elemento del conjunto, y si toda parte de un conjunto tiene primer elemento el conjunto se dice bien ordenado, sin preocuparnos, por ahora, de si todo conjunto ordenado puede ser o no ser bien ordenado.

4. El numero ordinal.-Consideremos el conjunto ordenado de varios hombres: Pedro, Juan, Luis, Francisco, Ruperto y Gabriel, por ejemplo, y el conjunto, también ordenado, de varias mujeres: Petra, Juana, Luisa, Francisca, Ruperta y Gabriela, y representemos sus nombres por sus iniciales mayúsculas y minúsculas, respectivamente, $\mathrm{y}$ por $\mathrm{H} \quad \mathrm{y} \quad \mathrm{M}$ sus conjuntos, escribiendo:

$$
\begin{aligned}
& \mathrm{H}=P, J, L, F, R, G, \\
& \mathrm{M}=p, j, l, f, r, g .
\end{aligned}
$$

Entre estos conjuntos podemos establecer una correspondencia tal que al primer elemento $P$ de $H$ le corresponda el primero $p$ de $M$ y que si a un elemento cualquiera $L$ de $\mathrm{H}$ le corresponde el elemento $l$ de $\mathrm{M}$, también se correspondan los siguientes respectivos $F \quad$ y $f$. Recordando la definición 2 esta correspondencia es biunívoca si el último elemento de $\mathrm{H}$ tiene como homólogo el úlimo de $\mathrm{M}$ y recíprocamente, y es unívoca o plurívoca en el caso contrario.

Considerando ahora el conjunto A de los animales perro, jaguar, león, faisán, ratón y gato, que representaremos por sus iniciales mayúsculas acentuadas, así :

$$
\mathrm{A}=P^{\prime} J^{\prime}, L^{\prime}, F^{\prime}, R^{\prime}, G^{\prime},
$$

entre los elementos de este conjunto A y los de H y M podemos establecer una correspondencia, biunívoca o no, tal que a cada elemento $F$ de $\mathrm{H}$ le corresponda un elemento $F^{\prime}$ de $\mathrm{A}$ y al $f$ de M también el $F^{\prime}$ de $A$, en cuyo caso diremos que los elementos $F, f \quad$ y $\quad F^{\prime}$ ocupan el mismo lugar en sus conjuntos respectivos.

De esta definición resulta que, dados varios conjuntos, se pueden agrupar todos sus elementos poniendo en cada grupo los elementos que ocupan el mismo lugar en cada conjunto.

Colocando, pues, en columna los elementos de los conjuntos que ocupan el mismo lugar, tenemos el siguiente cuadro:

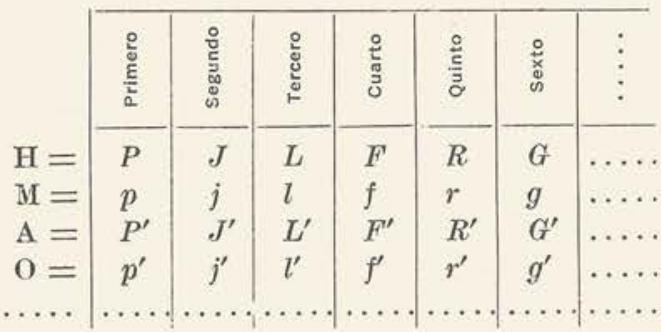

donde $\mathrm{O}$ es el conjunto de los objetos pluma-jarrolima-flor-remo-grifo, representados por sus iniciales minúsculas acentuadas, y los puntos suspensivos indican que el cuadro se puede prolongar vertical y horizontalmente cuanto queramos.

Agrupados así los elementos de los conjuntos dados, se llama número ordinal el ente abstracto que representa el conjunto de elementos que ocupan el mismo lugar en cada conjunto: primero, segundo, tercero, cuarto, etc.

5. El numero cardinal.-Supongamos que los hombres y mujeres que forman los conjuntos H y M antes considerados están en un salón y desean bailar. Es evidente que si cada hombre tiene una tocaya y sólo una y cada mujer tiene un tocayo y sólo uno y bailan por parejas homónimas, nadie se queda sin bailar; pero ¿ocurrirá lo propio si bailan dos personas de nombres distintos? En otros términos: ¿podrán bailar todos los hombres y todas las mujeres cualquiera que sea el modo de formarse las parejas? El sentido común nos contesta afirmativamente porque de una manera implícita estamos admitiendo que hay el mismo número de hombres que de mujeres.

Pero este número no es igual al estudiado antes. Los diferencia algo que conviene examinar. Si del salón en que suponemos reunidos los hombres y mujeres, se ausenta una de éstas: Luisa, por ejemplo, las personas restantes pueden bailar por parejas homónimas, excepto Luis, y si se aparean de otro modo, siempre se quedará un hombre sin bailar. En cambio, si se ausentan un hombre y una mujer sólo pueden ocurrir, en virtud del principio de contradicción, dos casos: que sean tocayos o que no lo sean. Si lo son, pueden bailar los tocayos restantes y nadie se quedará sin bailar, y si no lo son, porque se han marchado Petra y Francisco, por ejemplo, puede bailar Pedro con Francisca y los demás por parejas homónimas.

Pues bien, cuando entre un conjunto como el H y otro conjunto como el M existe una correspondencia buinívoca, se dice que ambos conjuntos son 
coordinables y es fácil demostrar no sólo el hecho antes observado de conservarse la coordinabilidad si se suprime un elemento de uno y otro del otro sino también esta propiedad fundamental: Si un conjunto es coordinable con otro de un cierto modo, también lo es de cualquier otro modo que se ensaye la coordinación.

Si consideramos ahora un elemento $A$ y ponemos a continuación el conjunto formado por este elemento $A$ y otro $B$; luego el $A B C$ que contiene el conjunto anterior y un nuevo elemento $C$; después el $A B C D$ constituído por el $A B C$ y un cierto elemento $D$, etc., tendremos la sucesión fundamental de conjuntos

$\begin{array}{ccccccc}\text { Uno } & \text { Dos } & \text { Tres } & \text { Cuatro } & \text { Cinco } & \text { Seis } & \ldots . \\ 1 & 2 & 3 & 4 & 5 & 6 & \\ A & A B & A B C & A B C D & A B C D E & A B C D E F & \ldots\end{array}$

y entonces todos los conjuntos de la Naturaleza se pueden clasificar colocando cada uno en el grupo encabezado por el de la sucesión fundamental con el cual es coordinable.

Por ejemplo, dado el conjunto de cosas silla-hilomesa-tintero - $\mathrm{s} \cdot \mathrm{h} \cdot \mathrm{m} \cdot \mathrm{t}$ - lo pondremos en el que está encabezado por el $A B C D$; los conjuntos de hom. bres, mujeres, animales y objetos que hemos considerado antes, los colocaremos en el que lleva como guión el $A B C D E F$; etc.

Clasificados así los elementos de los conjuntos, se llama número cardinal el ente abstracto que representa todos los conjuntos que son coordinables entre sí y los distingue de los no coordinables: uno, dos, tres, cuatro, etc. Decir, por tanto, que dos conjuntos son coordinables equivale a decir que tienen el mismo número cardinal o igual número de elementos, cualquiera que sea la naturaleza de éstos y el orden en que estén colocados.

6. Correspondencia entre los numeros ordinales x cardinales.-Vemos, pues, que hay una diferencia esencial entre los números ordinales y cardinales. El número ordinal 6 designa a Gabriel en el conjunto de hombres, a Gabriela en el de mujeres, al gato en el de animales y al grifo en el de objetos, es decir: Gabriel es el sexto hombre del conjunto H, el gato el sexto animal del conjunto A, etc., mientras que el número cardinal 6 representa el conjunto de todos los hombres, de todas las mujeres, de todos los animales y de todos los objetos, prescindiendo del orden en que estén colocados y de su naturaleza.

Ahora bien, a todo número ordinal, cuarto, por ejemplo, del conjunto $\mathrm{O}$ de objetos, o sea la flor, le corresponde el número cardinal cuatro que designa el número de elementos del conjunto plumajarro-lima-flor, y de todos los que, como él, son coordinables con el $A B C D$ de la sucesión fundamental y recíprocamente: disponiendo ésta y los conjuntos coordinables con cada uno de los de ella - conservando las siglas anteriores-en la siguiente forma:

\begin{tabular}{|c|c|c|c|c|}
\hline $1^{0}$ & $2^{9}$ & $4^{0}$ & $5^{\circ}$ & $6^{\circ}$ \\
\hline$A$ & $A B A B C$ & $A B C D$ & $A B C D E$ & $A B C D E F \ldots$ \\
\hline$\cdots$ & $\cdots \cdots$ & $s h m t$ & $\ldots \ldots$ & $\begin{array}{lllllll}P & J & L & F & R & G & \ldots \\
p & j & l & f & r & g & \ldots \\
P^{\prime} J^{\prime} L^{\prime} F^{\prime} & R^{\prime} G^{\prime} & \ldots \\
p^{\prime} & j^{\prime} & l^{\prime} & f^{\prime} & r^{\prime} & g^{\prime} & \ldots\end{array}$ \\
\hline
\end{tabular}

resulta que al número cardinal seis, por ejemplo, que representa el número de objetos del conjunto perro-jaguar-león-faisán-ratón-gato y de todos los que, como él, son coordinables con el $A B C D E F$ de la sucesión fundamental, le corresponde el número ordinal sexto del conjunto primero-segundo-tercerocuarto-quinto-sexto.

Por consiguiente, asociando a todo número ordinal $n_{o}$ su correspondiente cardinal $n_{c} \quad \mathrm{y}$ formando el conjunto de parejas

$$
\left(1_{\mathrm{o}} 1_{\mathrm{c}}\right),\left(2_{\mathrm{o}} 2_{\mathrm{e}}\right),\left(3_{\mathrm{o}} 3_{\mathrm{c}}\right),\left(4_{\mathrm{o}} 4_{\mathrm{c}}\right), \ldots,\left(n_{n} n_{c}\right), \ldots,
$$

se obtiene, por abstracción, el concepto de número natural como símbolo común de un número ordinal y su correspondiente cardinal, concepto que es ya un producto irreductible de la facultad creadora del espíritu humano que se ha dado en llamar "el demonio matemático", de tal modo que, como advierte Brunschvicg (León, 1869....., francés) "la noción de número corresponde a otra cosa que a un simple imperativo metodológico y tiene el valor de un indicativo metafísico. El principio del número significa no sólo que es preciso contar para comprender las cosas, sino que éstas tienen efectivamente una cuenta, están constituídas por partes más allá de las cuales no hay ya división y forman un todo pasado el cual no hay suma" (1).

Esta conquista, que hoy nos parece tan fácil, ha necesitado el transcurso de muchos siglos. Antes de ser nociones puras, los números fueron gestos en un orden ritual corpóreo, con un sentido antropomórfico $\mathrm{y}$ un valor concreto $\mathrm{y}$ cualitativo anterior a la idea cuantitativa que supone una suma, es decir: el número cardinal; y es preciso que avance la civilización para que la cualidad y la cantidad se yuxtapongan en una síntesis mental y el último término de una sucesión ordenada coincida con la unidad en bloque de un conjunto de objetos.

7. Principio de Schröder.-La síntesis mental aludida se comprueba experimentalmente cuando es pequeño el número de elementos de un conjunto; pero por poco crecido que sea dicho número es irrealizable la operación, lo que justifica la admisión del siguiente postulado fundamental de la Aritmética: El número de objetos de un conjunto es independiente del orden en que se cuenten, debido a Schröder (F. W. K., Ernst, 1841-1902, alemán) que lo enunció en su Lehrbuch der Aritmetik und Algebra, Leipzig, 1877.

En virtud de este postulado - llamado principio de invariación del número- podemos contar, es decir: numerar los elementos de un conjunto arbitra-

(1) Les étapes de la Philosophie nathénatique, París, 1912, pág. 348. 
riamente y siempre llegaremos al mismo resultado. En otras palabras: los conjuntos tienen un carácter invariante respecto de todas las ordenaciones posibles, y ese carácter es su número cardinal. Decir, pues, que el número de objetos de un conjunto es seis, por ejemplo, es lo mismo que decir que sus elementos se pueden numerar de uno a seis de cualquier manera $y$, por tanto, parece que el concepto de número cardinal es reductible lógicamente al de númeio ordinal, pero como aquél resulta de experiencias realizadas con conjuntos coordinables, independientes del número ordinal, no existe ningún lazo lógico de unión entre uno y otro, y por eso hemos dicho antes que dos conjuntos tienen el mismo número cardinal cuando se puede establecer una correspondencia biunívoca entre sus elementos, definición por abstracción que equivale a esta otra: Número cardinal es el carácter común a todos los conjuntos que se pueden numerar de la misma manera.

Este concepto, nacido de un hecho de experiencia generalizado, desborda el cuadro de las definiciones lógicas y para incluírlo en él Russell (Bertrand, A. W. $1872-\ldots$, inglés) ha dicho: El número de un conjunto es el conjunto de todos los conjuntos que son coordinables con él, úmica definición explícita de conjunto que se ha dado y que, como la de Cantor, tiene el inconveniente de emplear la palabra todos; pero considerada como dato del pensamiento, la definición de Russell nos dice que el concepto de número cardinal no se puede reducir, con exclusivos recursos lógicos, al concepto de número ordinal.

8. Conjuntos Finitos y conjuntos infinitos.Los conjuntos que nos han servido para construír el número natural son finitos, es decir: se pueden ordenar en el espacio o en el tiempo y tienen un primero y un último elemento. Su propiedad característica -demostrada por Dirichlet (Gustav P. Lejeune, 1805-1859, alemán) - es la de no ser coordinable con ninguna de sus partes, propiedad que no es válida para los conjuntos infinitos o sea para aquellos que carecen de último elemento.

Por ejemplo: el conjunto de los números natura. les es coordinable con el de los números pares, que es una parte de aquél, pues que contiene, además, los números impares. La correspondencia puede establecerse asignando como homólogo de cada número del primer conjunto su doble en el segundo y a cada uno de los de éste su mitad en aquél.

En esta propiedad fundó Dedekind (J. W. Ri(chard, 1831-1916, alemán) la distinción entre conjuntos finitos e infinitos en su famoso opúsculo Was sind und was sollen die Zahlen?, Braunsweig, 1872 .

El conjunto infinito más sencillo es la sucesión de números naturales de la que no hay que hacer ningúm misterio. Con palabras de Tannery (Jules, 1848-1910, francés): "Decir que la sucesión natural es infinita equivale a este hecho: después de cada número hay otro", bien entendido que la idea que implica esta frase no corresponde a una intuición sensible, sino a la oposición entre lo individual y lo universal, de tal modo que el número, en cuanto pluralidad, exige la distinción entre la existencia de cada objeto singular - componente individual -y la invariación- componente universal-que constituye la esencia numérica para cuya realización por los individuos existentes es necesario el acto creador de éstos.

El infinito cuyos elementos se dan sucesivamente es el infinito potencial que cae bajo el dominio de Ia Aritmética y aquel cuyos elementos se consideran simultáneamente es el infinito actual que estudia el Análisis y cuyo conocimiento no es un problema matemático sino filosófico.

Pero a la Matemática no le preocupa concebir el infinito actual porque, gracias a la creación cantoriana, sabe manejarlo, estableciendo un convenio cualquiera - siempre que no sea contradictoriotal que, dado un objeto lo incluímos en el conjunto si satisface el convenio o lo exclúmos de él si no lo satisface.

Esto no quiere decir, sin embargo, que podamos saber si un objeto dado pertenece o no pertenece al conjunto. El conjunto de los números algebraicos, por ejemplo, está definido sin ambigüedad y todavía no sabemos si el número $2^{\sqrt{2}}$ es algebraico o trascendente.

9. Construccion de la Aritmetica.-En posesión del número natural, con dos de ellos se construye el racional, con infinitos números racionales se crea el número real, con dos números reales se forma el complejo y con todos ellos la Aritmética: regina Mathematicae, con palabras de Gauss (J. F. Karl, 1777-1855, alemán), como ésta es la regina scientiarum.

Las sucesivas ampliaciones del concepto de número son de todos bien conocidas: una pareja de números naturales en un orden define el número racional como ente abstracto que permite representar los conjuntos de igual valor, es decir, los intercambiables desde un cierto punto de vista; una clasificación de los números racionales en dos grupos tales que todo número pertenezca a uno de los grupos y sólo a uno y que todo número de uno de los grupos sea menor que todo número del otro es una cortadura - creación de Dedekind-que nos da el número real, y, finalmente, de acuerdo con Hamilton (William Rowan, 1805-1865, inglés), todo par ordenado de números reales cualesquiera es un número complejo ordinario, último escalón de la Aritmética.

Estas distintas generalizaciones están impuestas por la necesidad de resolver problemas cada vez más complicados. La sustracción cuando el minuendo es menor que el sustraendo obliga a la creación del número negativo, la división si el dividendo no es múltiplo del divisor da origen al fraceionario que, con el negativo, componen el racional; la extracción 
de raíces de números no potencias perfectas hace preciso el número irracional que, con el racional, constituyen el real que amplía poderosamente el radio de acción de la Aritmética; pero como todavía quedan sin sentido algunas operaciones tales como las potencias de base racional y exponente irracional, los logaritmos de los números negativos, etc., se crea el número complejo en cuyo campo son ya posibles dichas operaciones.

A esta necesidad de tipo aritmético se une otra de carácter geométrico: la representación de los puntos a la derecha y a la izquierda de un punto tomado como origen de distancias y la de todos los puntos de la recta $\mathrm{y}$ del plano.

Ahora bien, a fin de que la Aritmética sea una ciencia no-contradictoria es preciso extender a cada nuevo sistema de números las operaciones fundamentales con sujeción al principio de permanencia de las leyes formales de Hankel (Hermann, 1839. 1873, alemán) : uniforme, asociativa, conmutativa, transitiva y distributiva, y como no existe ningún sistema de números complejos de más de dos componentes en que el producto satisfaga todas las leyes formales - propiedad que demostró Weierstrass (Karl, 1815-1897, alemán) en 1863 y constituye el llamado último teorema de la Aritmética- el desarrollo normal de esta ciencia termina con la creación de los complejos ordinarios.

10. SUMA Y PRoducto.-Las dos operaciones fundamentales de la Naturaleza son la yuxtaposición y la reproducción que se traducen en Aritmética por suma y producto, respectivamente.

Llamamos suma de dos conjuntos $\mathrm{M}$ y $\mathrm{N}$ que no tienen ningún elemento común, al conjunto formado por los elementos de M y de N, definición válida para la suma de conjuntos de conjuntos sin ningún elemento común considerados de dos en dos, y suma de dos números $m$ y $n$ es el número cardinal del conjunto-suma de dos conjuntos M y N cuyos números cardinales son $m$ y $n$ respectivamente.

Producto de dos conjuntos M y $\mathrm{N}$ es el conjunto formado por los pares de elementos obtenidos asociando cada elemento de M con cada elemento de $\mathrm{N}$ de todas las maneras posibles, y producto de dos números $m$ y $n$ es el número cardinal del conjunto-producto de dos conjuntos M y N cuyos números cardinales son $m$ y $n$ respectivamente.

Si, para fijar las ideas, suponemos que es

es

$$
\begin{aligned}
& \mathrm{M}=A, \quad B, \quad C, \\
& \mathrm{~N}=E, \quad F,
\end{aligned}
$$

$$
\text { II } \times \mathrm{N}=(A E),\left(A F^{\prime}\right),(B E),(B F),(C E),\left(C F^{\prime}\right),
$$

donde $A E, A F, \ldots, C F$, no son dos elementos sino el elemento obtenido asociando el $A$ con el $E$, el $A$ con el $F$, etc., como el hijo del Sr. Alvarez y de la Sra. Fernández es el Sr. Alvarez-Fernández: $u n$ ser y no dos.
Obsérvese que los productos $\mathrm{M} \times \mathrm{N}$ y $\mathrm{N} \times \mathrm{M}$ son distintos porque el Sr. Alvarez-Fernández es un ser distinto del Sr. Fernández-Alvarez, pero los productos $m n$ y $\mathrm{nm}$ son iguales como se demuestra sin más que establecer una correspondencia biunívoca entre los conjuntos $\mathrm{M} \times \mathrm{N}$ y $\mathrm{N} \times \mathrm{M}$ haciendo corresponder a cada Sx. Alvarez-Fernández del primero el Sr. Fernández-Alvarez del segundo, y recíprocamente.

Como en la definición de producto de dos conjuntos no hamos hecho la restricción de que sean distintos todos sus elementos, podemos hacer, en particular, el producto de un conjunto M por él mismo -que se nota $\mathrm{M}^{2}-\mathrm{y}$ obtendremos el conjunto die las parejas cuyo primero y segundo elemento pertenecen a M y, por tanto, si $\mathrm{M}$ es cl conjunto de los números reales, $\mathrm{M}^{2}$ es el de las parejas de números reales, o sea: el conjunto de los puntos de un plano.

11. Funcion.-Si en el coujunto de pares $(m, n)$, es decir: en el prodncto $\mathrm{M} \times \mathrm{N}$, consideramos un conjunto de pares $(m, n)$ tales que todo elemento de ií entre en un par y sólo en uno, pero pudiendo ciertos elementos de $\mathrm{N}$ figurar en varios pares $\mathrm{y}$ otros no figurar en ninguno, e imaginamos con Possel (René, 1905-...., francés) los dos elementos de cada par unidos por un hilo, resulta que de cada elemento de M sale un hilo, y sólo uno, que termina en un elemento de $\mathrm{N} y$, por tanto, a un elemento cualquiera $m$ de $\mathbf{M}$ se encuentra asociado un elemento $n$ de $\mathrm{N}$ que se representa por $m(n)$.

Hemos definido, pues, una función generalizada o aplicación en la que $m$ es el valor de la variable y $n$ el valor correspondiente de la función, llamado también imagen de $m$. Por consiguiente, dado un subconjunto $\mathbf{M}^{\prime}$ de $\mathbf{M}$, el conjunto de las imágenes de los elementos de $\mathrm{M}^{\prime}$ se llama imagen de $\mathrm{M}^{\prime}$ y encontramos el concepto general de función cuando M y N son conjuntos de nú. meros: Se dice que el número y es función del número $\mathrm{x}$ que varía en un campo dado $\mathrm{C}$ cuando a todo número $\mathrm{x}$ dado en $\mathrm{C}$ corresponde por una ley determinada pero de naturaleza y expresión completamente arbitrarias, un número y y sólo uno, concepto debido a Riemann (Bernhard, 18261866, alemán) que comprende como caso particular el de Euler (Leonard, 1707-1783, suizo), ya derogado.

Si todo elemento de $\mathrm{N}$ figura también en un par, y sólo en uno, queda definida una transformación o correspondencia biunívoca entre M y N, de modo que los papeles de la variable y de la función son intercambiables y podemos decir que también hemos definido una transformación de $\mathrm{N}$ en $\mathrm{M}$.

12. Producto de varios factores.-La definición anterior de producto de dos factores equivale a asociar a dos signos distintos $\mu \quad y \quad v \quad$ dos entes abstractos $m$ y $n$, distintos o no, para construír el par $(m, n)$. Este concepto fundamental puede ge- 
neralizarse fácilmente sustituyendo los signos $\mu$ y $v$ por un conjunto $\mathrm{C}=(a, b, c, \ldots l, \ldots) \mathrm{y}$ asociando a todo elemento de $\mathrm{C}$ un ente $\alpha_{\mathrm{i}}$, siendo $\operatorname{los} \alpha_{i}$ distintos o no. Si a todo elemento $l$ de $\mathrm{C}$ le asociamos un conjunto $\mathrm{A}_{i}$, siendo $\operatorname{los} \Lambda_{\mathrm{i}}$ distintos o no y con elementos comunes o no, llamaremos producto de los conjuntos

$$
A_{a}, A_{b}, A_{c}, \ldots, A_{1}, \ldots
$$

según el conjunto C, el conjunto de todas las agrupaciones

$$
\left(\alpha_{n}, \alpha_{b}, \alpha_{c}, \ldots, \alpha_{1}, \ldots\right)
$$

siendo $\alpha_{1}$ un elemento de $A_{1}$, de donde resulta que si $\quad \mathrm{C} \quad$ y $\quad \mathrm{A}_{i}$ son finitos, el número de elementos del conjunto-producto es el producto de los elementos de los $A_{i}$.

13. Exponenciacion:-Un caso particular notable es aquel en que todos los $A_{i}$ son iguales a un conjunto A. Las agrupaciones se obtienen entonces asociando a todo elemento de $\mathrm{C}$ un cierto elemento de A y el conjunto de todas ellas, es decir: el producto de los conjuntos A según el conjunto C se llama exponencial de base A y exponente $\mathrm{C} \quad \mathrm{y}$ se representa por $\mathrm{A}^{\mathrm{c}}$. Es claro que si A y C son finitos y sus números cardinales son a y $c$, respectivamente, el número de elementos de $\mathrm{A}^{c}$ es $a^{c}$.

De aquí se deduce una consecuencia muy importante. Si C es el conjunto de los números natura. les, todo elemento de $\mathrm{A}^{\mathrm{c}}$ es la función que se obtiene asociando a cada número natural $n$ un elemento $a_{n}$ de $\mathrm{A}$, es decir: una sucesión de elementos de A. Por ejemplo: si A se compone de los diez elementos $0,1,2,3,4,5,6,7,8,9$, toda sucesión

$$
\begin{gathered}
\mathrm{C}=0,1,2,3,4,5,6,7,8,9,10,11,12, \ldots . \\
\mathrm{A}^{\mathrm{c}_{\mathrm{i}}}=2,7,4,0,2,4,9,1,5,6,7,2,8, \ldots .
\end{gathered}
$$

se puede considerar como la parte decimal del desarrollo ilimitado en el sistema de numeración de base 10 , de un número real $x$ - en este caso es $x=0,2740249156728 \ldots$ - tal que es $0 \equiv x \equiv 1, \quad y$ $\mathrm{A}^{\mathrm{c}}$ es el conjunto de los números reales, que se representa por $\mathfrak{C}$, algunos de los cuales están repetidos, como por ejemplo: $0,37=0,369999 \ldots$, porque siendo, por definición $0,999 \ldots=1$, toda fracción decimal de un número limitado de cifras se puede sustituír por la que resulta de disminuír la última cifra en una unidad y agregarle infinitos nueves, lo que nos da un medio de construír el conjunto de los números reales partiendo del conjunto de los números naturales.

Si A y C son ambos el coujunto de los nú. meros reales, todo elemento de $A^{c}$ es una función definida por la asociación de todo número real a un número real, es decir: una función de variable real, y $A^{c}$ será el conjunto de todas las funciones de variable real.

Si A sólo tiene dos elementos a y $b$, toda agrupación del conjunto $2^{\mathrm{c}}$ se consigue asociando a unos elementos de $\mathrm{C}$ el elemento $a$ y a otros el $b \quad$ y tenemos una función definida en el conjunto $\mathrm{C}$ que sólo toma dos valores, $\mathrm{y}$, por tanto, toda agrupación es una separación de C en dos partes: la que tiene sus elementos asociados a $a$ y la que los tiene asociados a $b$ y el conjunto $2^{c}$ es entonces el conjunto de todos los subconjuntos de C, incluyendo el propio conjunto $\mathrm{C}$ y el conjunto nulo.

\section{EL CONTINUO}

14. Conjuntos numerables.-Un concepto fundamental de la teoría de conjuntos es el de numerabi. lidad o posibilidad de establecer una correspondencia biunívoca entre la sucesión natural y los elementos de un conjunto, lo que equivale a poder enumerar éstos en un cierto orden, o, lo que es lo mismo: dar los elementos de un conjunto sucesivamente, lo que no quiere decir que el conjunto está dado cuando, establecida una ley, se pueden determinar todos los elementos de un conjunto porque ello equivaldría a reducir el infinito potencial al infinito actual.
Escribamos los números racionales en un cuadro formado del siguiente modo: En la primera fila ponemos los enteros, que equivalen a fracciones de denominador 1 , ordenados de menor a mayor; en la segunda fila las fracciones de denominador 2 ordenadas de igual manera, en la tercera las de denominador 3 , etc., y luego de tachar las fracciones reducibles que aparecen escritas dos veces - por ejem. plo: la $4 / 8$ que está en la octava fila y equivale a $1 / 2$ que figura en la segunda- resulta:

$$
\begin{aligned}
& \ldots-4-3-2-1+1+2+3+4+5 \ldots \\
& \ldots-7 / 2-5 / 2-3 / 2-1 / 2+1 / 2+3 / 2+5 / 2+7 / 2+9 / 2 \ldots \\
& \ldots-5 / 3-4 / 3-2 / 3-1 / 3+1 / 3+2 / 3+4 / 3+5 / 3+7 / 3 \ldots \ldots \\
& \text {... }-7 / 4-5 / 4-3 / 4-1 / 4+1 / 4+3 / 4+5 / 4+7 / 4+9 / 4 \ldots
\end{aligned}
$$


Empezando por +1 y siguiendo el orden indicado en el esquema

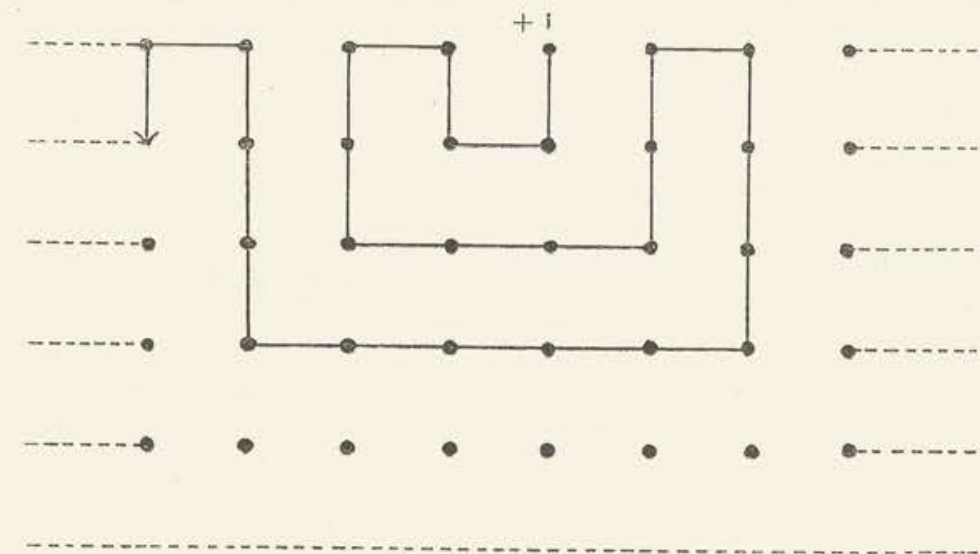

se obtiene la sucesión ordenada

$$
\underbrace{1}_{+1} \underbrace{2}_{+1 / 2} \underbrace{3}_{-1 / 2} \underbrace{4}_{-1} \underbrace{5}_{-2} \underbrace{6}_{-3 / 2} \underbrace{7}_{-2 / 3} \underbrace{8}_{-1 / 3} \underbrace{9}_{+1 / 3} \underbrace{10}_{+2 / 3} \underbrace{1}_{+3 / 2} \ldots
$$

lo que nos dice que el conjunto de los números racionales es coordinable con el de los números naturales.

Estos conjuntos infinitos se llaman numerables y su propiedad fundamental es: Todo conjunto de conjuntos numerables es un conjunto numerable.

Consideremos, en primer lugar, dos conjuntos numerables

$$
\begin{aligned}
& \mathrm{A}=a_{1}, a_{2}, a_{3}, \ldots \\
& \mathrm{B}=b_{1}, b_{2}, b_{3}, \ldots
\end{aligned}
$$

Se ve inmediatamente que si ordenamos los elementos de los conjuntos A y B así:

$$
\underbrace{1}_{a_{1}} \underbrace{2}_{b_{1}} \underbrace{3}_{a_{2}} \underbrace{4}_{b_{3}} \underbrace{5}_{a_{3}} \underbrace{6}_{b_{3}} \ldots
$$

el conjunto formado por todos los elementos de A y $B$ es numerable.

De análoga manera se demuestra que la propiedad subsiste cuando los conjuntos son dos, tres, cuatro, etc., $\mathrm{y}$, en general, cuando es finito el número de conjuntos.

Si son

$$
\begin{aligned}
& \mathrm{A}=a_{1}, a_{2}, a_{3}, a_{4}, \ldots ., \\
& \mathrm{B}=b_{1}, b_{2}, b_{3}, b_{4}, \ldots \ldots, \\
& \mathrm{C}=c_{1}, c_{2}, c_{3}, c_{4}, \ldots \ldots, \\
& \mathrm{D}=d_{1}, d_{2}, d_{3}, d_{4}, \ldots \ldots, \\
& \ldots \ldots \ldots . . . \ldots \ldots,
\end{aligned}
$$

infinitos conjuntos numerables, colocándolos uno debajo de otro, se pueden numerar ordenándolos a partir del elemento $a_{1}$ y siguiendo la dirección indicada por las flechas de la figura de enfrente, se tiene:

$$
\underbrace{1}_{a_{1}} \underbrace{2}_{a_{2}} \underbrace{3}_{b_{1}} \underbrace{4}_{a_{3}} \underbrace{5}_{b_{2}} \underbrace{6}_{c_{1}} \underbrace{7}_{a_{4}} \underbrace{8}_{b_{3}} \underbrace{9}_{c_{2}} \underbrace{10}_{d_{1}} \underbrace{11}_{a_{5}} \ldots
$$

Esta propiedad pone de manifiesto la importancia de los conjuntos numerables y podría servir para es- tablecer la línea divisoria de la Aritmética y la Geometría, estudiando la primera los conjuntos finitos y el infinito potencial y la segunda el continuo y el ultracontinuo; pero, como veremos, existen conjuntos infinitos que no son numerables y caen bajo el dominio de la Aritmética.

Del teorema fundamental que acabamos de demostrar se deduce inmediatamente:

I. Toda parte de un conjunto numerable es numerable.

II. Si los elementos de un conjunto se pueden poner en correspondencia biunívoca con los de un conjunto numerable, es numerable.

III. Todo conjunto cada uno de cuyos elementos se puede determinar por un número finito de subindices que toman valores naturales, es numerable.

15. Potencia de un conjunto.-Para comparar dos conjuntos finitos desde el punto de vista numérico, asociamos un elemento de uno a un elemento del otro hasta agotar los elementos de uno de los conjuntos, formando así un conjunto de parejas que definen una transformación de un conjunto A en otro B.

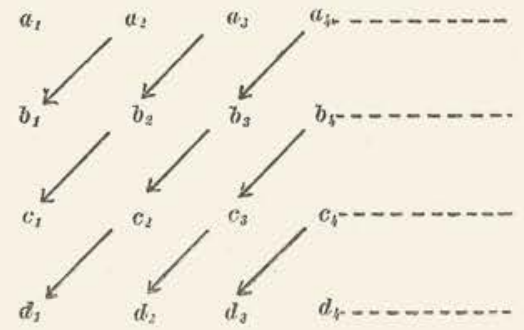

Parece natural emplear un proceso análogo cuando se trata de dos conjuntos infinitos, y esto se logra estableciendo entre ellos una transformación. 
Si ésta existe, decimos que ambos conjuntos son equivalentes, o que tienen la misma potencia.

El interés de este concepto, introducido por Cantor (1), radica en la existencia de conjuntos que tienen distintas potencias, $\mathrm{y}$ desempeña, por tanto, en los conjuntos infinitos, el mismo papel que el nú. mero en los finitos; pero hay que advertir que la potencia toma del número el aspecto cardinal y no del ordinal.

De los conjuntos numerables, es decir: de los conjuntos cuyos elementos se pueden poner en correspondencia biunívoca con los números naturales, se dice que tienen la potencia del numerable que Cantor representó por la primera letra $\boldsymbol{N}_{0}$ del ale. fato inicial de la palabra alemana abzählbar: numerable, afectada del subíndice 0 .

16. Continuo fisico y continuo matematico.Todo conjunto numerable se puede representar grá: ficamente por puntos alineados $y$ separados por intervalos de cualquier magnitud finita; pero como en un intervalo finito hay infinitos puntos individuales, para llenar el intervalo es preciso admitir un punto dotado de la propiedad de desplazamiento que le permita hacer desaparecer las individualidades puntuales, que es lo que constituye la esencia de la continuidad.

¿Puede conseguirse lo continuo por medio de lo numerable? Desde el punto de vista físico el problema está resuelto puesto que dos valores que caigan en el umbral de la sensación son iguales. Como es bien sabido, sólo cuando una luz, un sonido, un peso llega a una cierta cantidad percibimos algo, y la Psicología experimental dispone hoy de instrumentos adecuados para determinar qué aumento debe tener un excitante ya percibido para que se advierta una diferencia al percibirlo de nuevo. En el sonido, cuando oímos, por ejemplo, un acorde de quinta: do-sol, percibimos algo que tiene una cualidad perfectamente determinada y no los dos conjuntos de vibraciones que producen el do y el sol, y que dan origen a la quinta, porque la razón de ambas frecuencias $3 / 2$ se verifica en un tiempo que corresponde al mínimo audible y durante el cual se produce un número de trenes de ondas tal que las frecuen. cias vibratorias son muy pequeñas respecto de dicho número; en el tacto, el umbral es muy variable, pues mientras en la punta de la lengua es de $1 \mathrm{~mm}$. en la yema de los dedos es de $2 \mathrm{~mm}$. $y$ en las extremidades llega hasta $6 \mathrm{~cm}$., y en cuanto a la medición de segmentos, los físicos han logrado una precisión sorprendente en la longitud de onda de la radiación roja del cadmio que es del orden $10^{-6} \mu$.

Claro es que el nmbral de la sensación varía de unas personas a otras; pero existe un promedio fijo

(1) Über einen Satะ aus der Theorie stetigen Mannigfaltigkeiten, Götting. Nachrichten, 1879, pág. 119. Más tarde dice el mismo Cantor: "He tomado la palabra potencia de J. Steiner, que la emplea en un sentido especial para decir que dos figuras están relacionadas proyectivamente cuando a todo elemento de una le corresponde uno y sólo uno en la otra. En mi concepto de potencia se conserva la correspondencia biunivoca, pero, en cambio, no se impone ninguna restricción a la ley de coordinación". Öber unendliche lineare Punktmannigfaltigkeiten. Math. Ann., vol. XX, 1882, pág. 183. que nos permite dar por resuelto el problema de la continuidad física, $\mathbf{y}$, así por ejemplo, un peso $a$ de $10 \mathrm{gr}$. y un peso $b$ de 11 gr. producen sensaciones idénticas, como también el peso $b$ de $11 \mathrm{gr}$. y uno $c$ de $12 \mathrm{gr}$.; pero las sensaciones que producen los pesos de $10 \mathrm{y}$ de $12 \mathrm{gr}$. son distintas, resultados experimentales que nos permiten escribir $a=b, b=c, a<c$ que constituye según Poincaré (Henri, 1854-1912, francés) la fórmula del continuo físico.

Y como esta fórmula denuncia un desacnerdo intolerable con el principio de contradicción, ha habido necesidad de inventar el continuo matemático inalcanzable por medio de la numerabilidad, como reconoció el propio Cantor al invocar un nuevo principio para pasar de to numerable a lo continuo. Lo numerable es disyuntivo, lo continuo copulativo, y tienden a la separación y a la reunión respectiva. mente; lo numerable es un intervalo espacial o temporal, lo continuo es nin devenir que pasa de lo vir. tual a lo efectivo.

17.-Postulado de continuidad De la Recta.La existencia en una recta de puntos de abscisa irracional exige la recíproca para la construcción lógica de la Geometría, lo que se consigue con el postu. lado de continuidad de la recta que tiene varias formas, la más sencilla de las cuales es la de Dedekind:

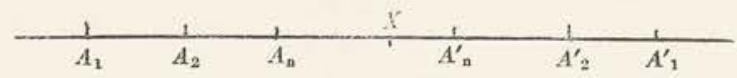

Si los segmentos $A_{1} A_{1}^{\prime}, A_{2} A_{2}^{\prime}, \ldots, A_{\mathrm{n}} A_{\mathrm{n}}^{\prime}$ for man una sucesión decreciente y cada uno está contenido en el anterior, existe un punto $\mathrm{X}$ situado en todos ellos, y no puede haber otro punto $X^{\prime} \ddagger X$ porque entonces serían todos los segmentos $A_{\mathrm{i}} A^{\prime}{ }_{\mathrm{i}}$ mayores que $X X^{\prime}$, contra la hipótesis.

Este punto único $X$ se toma como correspondiente al número real $x, y$ procediendo así con todos los números reales queda establecida una correspondencia biunívoca entre el conjunto de los números reales $\mathrm{y}$ el conjunto de los puntos de la recta.

Este postulado es el fundamento de la Geometría Analítica que, al hacer corresponder a cada punto de la recta una abscisa y a cada número real un punto en la recta, considera sinónimas las palabras punto y número y al sustituír los puntos por sus números correspondientes, opera con éstos y traduce después los resultados al lenguaje geométrico.

Las magnitudes cuyas cantidades se pueden poner en correspondencia biunívoca con los números reales, se llaman continuas.

18. Potencia del contrnuo.-Antes de abordar el problema de la numerabilidad del conjunto de los números reales, demostremos este hecho sorprendente: El conjunto de todos los números reales tiene la misma potencia que el de los comprendidos entre 0 y 1.

Para ello imaginemos representado el conjunto de todos los números reales sobre una recta $X Y$ 
dividida en dos semirrectas por el origen $O$ de abscisas y el conjunto de los números reales

$$
0 \equiv x \equiv 1
$$

en el segmento-unidad $A B$ perpendicular a $X Y$ tal que sea $O A=O B=1 / 2$. Si por los puntos $A \quad$ y donde $a, b, c, \ldots$ son las cifras $0,1,2, \ldots, 9$ en un orden y disposición cualquiera, $\mathrm{y}$ para evitar todo equívoco convendremos en emplear exclusivamente fracciones decimales de un número ilimitado de cifras (13) suponiendo por tanto, que las frac-

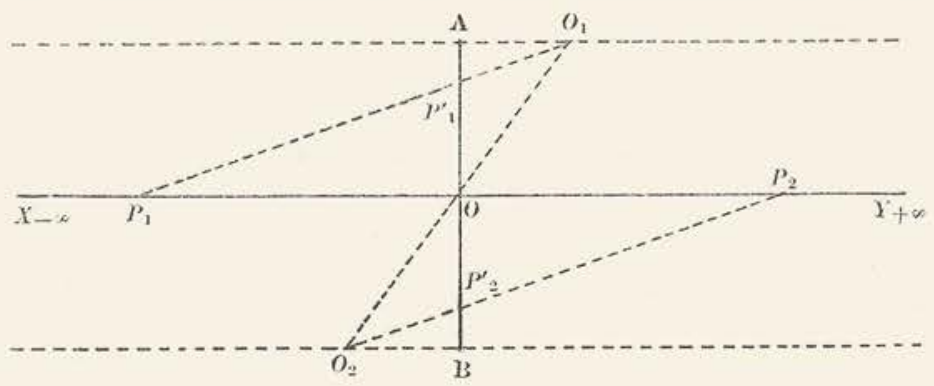

$B$ trazamos sendas paralclas a $X Y$ y tomamos en ellas dos puntos $O_{1}$ y $O_{2}$ simétricos respecto de $O$, resulta que a cada punto $\left\{\begin{array}{l}P_{1} \\ P_{2}\end{array}\right\}$ de la semirrecta $\left\{\begin{array}{l}O X \\ O Y\end{array}\right\}$ le corresponde el $\left\{\begin{array}{c}P^{\prime}{ }_{1} \\ P_{2}^{\prime}\end{array}\right\}$ del segmento $\left\{\begin{array}{l}O A \\ O B\end{array}\right\}$ en que lo corta la recta $\left\{\begin{array}{l}O_{1} P_{1} \\ O_{2} P_{2}\end{array}\right\} \mathbf{y}$, recíprocamente, a cada punto $\left\{\begin{array}{l}P_{1}^{\prime} \\ P_{2}^{\prime}\end{array}\right\}$ del segmento $\left\{\begin{array}{l}O A \\ O B\end{array}\right\}$ le co. rresponde el punto $\left\{\begin{array}{l}P_{1} \\ P_{2}\end{array}\right\}$ de la semirrecta $\left\{\begin{array}{l}O X \\ O Y\end{array}\right\}$ en que la corta la recta $\left\{\begin{array}{l}O_{1} P_{1}^{\prime} \\ O_{2} P_{2}^{\prime}\end{array}\right\}$ y, en particular, al punto $O\left\{\begin{array}{l}\text { del segmento } A B \\ \text { de la recta } X Y\end{array}\right\}$ le corresponde el mismo punto $\left\{\begin{array}{l}\text { de la recta } X Y \\ \text { del segmento } A B\end{array}\right\}$ y al $\left\{\begin{array}{c}A \\ B\end{array}\right\}$ el $\left\{\begin{array}{l}X_{-\infty} \\ Y+\infty\end{array}\right\}$ de la recta y recíprocamente. Siendo, pues, la correspondencia punto a punto, o número a número, en virtud del postulado de continuidad de la recta, es decir: binnívoca sin excepción, queda demostrado el teorema que creeríamos falso si, dejándonos llevar de la intuición, afirmásemos que una recta tiene más puntos que un segmento porque es mayor que éste, lo que nos advierte los peligros de creer válidos para los conjuntos infinitos conceptos y propiedades sólo demostradas para los conjuntos finitos.

Vemos, pues, que el conjunto de los números reales lo podemos sustituír por los números reales del segmento-unidad.

El problema de la numerabilidad del conjunto real to abordó Cantor por el método de las diagonales que da para toda sucesión numerable imaginada de todos los números reales un número real que no figura en ella, lo cual es una contradicción que demuestra que el conjunto $\mathfrak{U}$ no es numerable.

Escribamos, en efecto, todos los números reales de $\mathfrak{C}$ en forma de fracción decimal y supongamos que están en una sucesión numerable

$$
\begin{aligned}
& x_{1}=0, a_{1} a_{2} a_{3} \ldots . \\
& x_{2}=0, b_{1} b_{2} b_{3} \ldots . \\
& x_{3}=0, c_{1} c_{1} c_{3} \ldots . \\
& \ldots \ldots . . . . . . . .
\end{aligned}
$$

ciones anteriores tienen infinitos nueves a partir de una cierta cifra.

Si imaginamos ahora la diagonal que pasa por las cifras $a_{1}, b_{2}, c_{3} \ldots$ y formamos un número decimal $x^{\prime}$ que tenga por primera cifra una $a_{1}^{\prime} \ddagger a_{1}$, por segunda una $b_{2}^{\prime} \neq b_{2}$, por tercera una $c_{3}^{\prime} \neq c_{3}$, etc., este número

$$
x^{\prime}=0, a_{1}^{\prime} b_{2}^{\prime} c_{c}^{\prime} \ldots
$$

es distinto de todos los $x$, e incluso podemos suponer que todas sus cifras son diferentes de 0 y de 9 si queremos excluír del segmento-unidad sus extre$\operatorname{mos} 0$ y 1.

Es $\quad x^{\prime} \ddagger x$ porque sus primeras cifras $a_{1}$ y $a_{1}^{\prime}$ son distintas $\mathbf{y}$ dos fracciones decimales ilimitadas sólo pueden ser iguales cuando son iguales las cifras del mismo orden; análogamente es $x^{\prime} \neq x_{2}$ por ser $b^{\prime} \ddagger b_{2}$, etc.; luego la existencia del número $x^{\prime}$ distinto de todos los $x$ demuestra que cl conjunto de los números reales no es numerable, $y$ al no ser coordinable el conjunto $1,2,3, \ldots, n, \ldots$. de todos los números naturales y el de todos los números reales comprendidos entre 0 y 1 , tenemos dos potencias distintas: la de los conjuntos numerables que tienen la potencia \$ y la de los números reales del intervalo 0-1 que tienen la $\mathbb{C}$, llamada potencia del continuo.

19. Comparacion de infinitos.-Estamos, pues, en presencia de dos infinitos: el conjunto de todos los puntos de una recta y el conjunto de todos los puntos de abscisa racional, y, por consiguiente, parece que podemos pensar conjuntos infinitos distintos y también compararlos.

La teoría de conjuntos gira en la órbita de este problema, cuya solución se funda en una observación hecha por Bolzano (Bernhard, 1781-1848, checo) a propósito de comparar no dos conjuntos infinitos sino uno finito y otro infinito (1), cuya diferencia esencial es, como sabemos $(8)$, que, dado un conjunto infinito, siempre podemos encontrar una parte de él tal que a iodo elemento del subconjunto le corresponde un elemento del conjunto, observa-

(1) Paradoxien des Unendlichen, ed. póstuma de F. Prihonsky, Leipzig, 1851; $2^{a}$ ed. Berlin, 1899; $3^{a}, 1920$. 
ción elevada después a la categoría de teorema por Dirichlet, y que puede servir para comparar los infinitos si consideramos como equivalentes en el sentido cantoriano (15) los conjuntos que se corresponden elemento a elemento, y entonces si los conjuntos M y $\mathrm{N}$ no son equivalentes $y$ tienen por potencias $\mu$ y $v$, ipodemos decir que una de estas dos potencias es mayor que la otra?

Volvamos un momento a los conjuntos finitos $y$ pensemos, para fijar las ideas, los conjuntos de personas y de sillas que hay en un salon, y supongamos que una persona se sienta en una silla, luego una segunda persona en una segunda silla y así sucesivamente hasta que se agote uno de los conjuntos. Si el de personas se agota antes que el de sillas, el número de personas es menor que el de sillas, de modo que dados dos conjuntos finitos M y N cuyos númeres cardinales son $m$ y $n$, si if es equivalente a una parte de $\mathrm{N}$, es $m<n$.

Pero si los conjuntos M y N son infinitos, el probiema ya no es taì sencillo. Hemos visto que el conjunto de los números naturales es coordinahe con al de los números pares, que es un subconjunto de él; más aún: qne el conjunto racional, que es una parte del natural, es equivalente a éste, y es fácil demostrar el siguiente teorema: Si de un conjunto infinito se suprime un conjunto infinito $n$ merable, el conjunto formado por los elementos no suprimidos es un conjunto infinito que tiene la mis. ma potencia que el primitivo.

Si es A el conjunto infinito dado y B el conjunto infinito numerable que suprimimos, es

$$
\mathrm{A}=\mathrm{B}+\mathrm{A}^{\prime} \text {. }
$$

Este conjunto $\mathrm{A}^{\prime}$ es infinito porque el $\mathrm{B}$ se ha formado suprimiendo un primer elemento, luego un segundo, después un tercero, etc., sin agotar A porque si lo hubiéramos agotado sería finito; luego también podemos poner $\mathrm{A}^{\prime}=\mathrm{B}^{\prime}+\mathrm{A}^{\prime \prime}$, siendo $\mathrm{B}^{\prime}$ numerable y $\mathrm{A}^{\prime \prime}$ infinito. Ahora bien, como el conjunto $\mathrm{B}+\mathrm{B}^{\prime}$ de dos conjuntos numerables es un conjunto numerable $B^{\prime \prime}$, es $A=B^{\prime \prime}+A^{\prime \prime}$ y como $B^{\prime \prime} \quad$ y $B^{\prime}$ tienen la misma potencia porque ambos son numerables y A y $A^{\prime}$ se pueden considerar como conjuntos de los conjuntos $\mathrm{B}^{\prime \prime}$ y $B^{\prime}$ y el conjunto $A^{\prime \prime}$, resulta que $A$ y $A^{\prime}$ tienen igual potencia.

De aquí se deduce esta importante propiedad: La potencia del numerable es la menor potencia de los conjuntos infinitos.

Por último, enunciemos sin demostración, porque es larga y complicada, el llamado teorema de equivalencia debido a Bernstein (Félix, 1878...., alemán): Si M es equivalente a una parte de $\mathrm{N}$ y $\mathrm{N}$ equivalente a una parte de $\mathrm{M}$, los conjun. tos M y N son equivalentes, el cual puede considerarse como caso particular del teorema de Banach: Si la función $\varphi$ transforma de una mane. ra binnivora el conjunto A en un subconjunto B $y$, de igual modo, la función $\psi$ transforma
$\mathrm{B}$ en un subconjunto de A, existe una descom. posición de los conjuntos A y $\mathrm{B}$,

$$
\mathrm{A}=\mathrm{A}_{1}+\mathrm{A}_{2}, \quad \mathrm{~B}=\mathrm{B}_{1}+\mathrm{B}_{2}
$$

que satisface las condiciones

$$
A_{1} A_{2}=0, \quad B_{1} B_{2}=0, \quad \varphi(A)=B_{1}, \psi\left(B_{2}\right)=A_{2},
$$

cuya demostración puede verse en los Fundamenta Mathematicae, vol. VI, Varsovia, 1924, pág. 236.

El teorema de Berustein establece la siguiente definición :

Si M y N son dos conjuntos cualesquiera de potencias respectivas $\mu \quad$ y $v$ y lente a una parte de $\mathrm{N}$ pero no a $\mathrm{N}$, se dice que $\mu$ es menor que $v$ o que $v$ es mayor que H. escribiendo $\mu<\begin{array}{lll}v & o & v>\mu \text {. }\end{array}$

Claro es que cuando los conjuntos son finitos estas desigualdades son las que se verifican entre los números naturales.

Tenemos, pues, los siguientes casos:

I. Si M es equivalente a $\mathrm{N}$, es

$$
\mu=v \quad \text { y } \quad v=\mu
$$

por definición.

II. Si M es equivalente a una parte de $\mathrm{N}$ y $\mathrm{N}$ equivalente a una parte de $\mathrm{M}$, es

$$
\mu=v \quad \text { y } \quad v=\mu
$$

en virtud del teorema de Bernstein.

III. Si M es equivalente a una parte de $\mathrm{N}$ y $\mathrm{N}$ no es equivalente a ninguna parte de $M$, es $\mu<v \quad$ y $\quad v>\mu$.

IV. $\mathrm{Si} \mathrm{N}$ es equivalente a una parte de M $\mathrm{y}$ M no es equivalente a ninguna parte de $\mathrm{N}$, es $v<\begin{array}{lll}\mu & \text { y } & \mu\end{array}>v$.

V. Si M no es equivalente a ninguna parte de $\mathrm{N}$ ni $\mathrm{N}$ equivalente a ninguna parte de $\mathrm{M}$, no se puede concluír nada.

Hasta ahora no se ha encontrado ningún ejemplo que ilustre este último caso. Más adelante, cuando estudiemos el axioma de elección, abordaremos la posibilidad de la imposibilidad de que se presente. Cantor no sólo admite que también es $\mu=v$ sino que parece postular que los conjuntos M y N deben ser finitos $\mathrm{y}$, por tanto $\mu$ y $v$ números cardinales finitos y ordinales iguales.

La demostración de este último resultado preocupa actualmente a los matemáticos para poder justificar las definiciones de las operaciones con conjun. tos infinitos y, por consiguiente, con potencias.

20. Continuidad y discontinuidad.-Obsérvese que estos conjuntos infinitos de cuya comparación hemos hablado, son infinitos actuales, es decir: infinitos cuyos elementos hay que concebir simultáneamente todos, no los infinitos potenciales cuyos elementos se dan sucesivamente, que son los que maneja, en realidad, la Aritmética pues que todos los algoritmos infinitos que eaen bajo su jurisdicción están formados por un número finito $n$ de elementos y lo que hace es tomar $n$ lo suficiente- 
mente grande para que se cumplan ciertas condiciones.

Los infinitos que hemos comparado no son un devenir, sino un ser, o, empleando términos aristotéli$\cos$, son actos y no potencias, para no incurrir en el vicio de los logísticos enando consideran como infinitos actuales los infinitos eternos de que habla Pas(al (Blaise, 1623-1662, francés) que no son otra cosa sino indefinidos inmovilizados que justifican la opinión de Bloch (André,...., francés) sintetizada en una frase semejante al conocido apotegma escolástico y que, lanzada en 1926 , se ha hecho ya famosa: Nihil est in infinito quod prius non fuerit in finito (1), que es el grito de guerra de los finitistas actuales, y que conviene examinar porque en todas las cuestiones en que interviene el infinito - "nuestro amigo más amado, pero también el mayor enemigo de nuestra paz espiritual", según la bella frase de Pierpont (James..., norteamericano), apud Bull. Soc. Am. Math., 1928 - es indispensable precisar el sentido de cada vocablo para no caer en las redes del paralogismo.

La ley conmutativa de la suma nos autoriza a alterar arbitrariamente el orden de los sumandos sin que cambie el resultado, pero esta ley no es válida para ciertas series numéricas como las convergentes no absolutamente convergentes, es decir: las que convergen sin que converja la serie formada por los valores absolutos de sus términos, tal la bien conocidia

$$
1-1 / 2+1 / 3+1 / 4-1 / 5+\ldots .
$$

a la que se le puede asignar un valor arbitrario. En esto consiste lo que Bouligand (Georges,...., francés) ha llamado manifestación específica de la presencia de infinitos términos en el fenómeno de la dispersión de sumas (1); pero obsérvese que tal valor arbitrario es susceptible de la aproximación que queramos tomando un número finito de términos siempre que podamos elegir a voluntad un cierto número de términos positivos y otro cierto número de términos negativos y, por tanto, la afirmación de Bloch es legítima en cuanto el resultado de un pro-

(1) Les fonctions holomorphes et méromorphes dans le cercle-tinité, Mémorial des Sciences mathématiques, fasc. XX, París, 1926.

(2) Le finitisme et son efficacité dans la recherche mathèmatique, Revue Scientifique, vol. LXVI, pág. 585, París, 1928. blema infinito proviene de la superposición de un conjunto de problemas finitos.

Esto demuestra al propio tiempo el paralelismo que existe entre la Física y la Matemática contemporáneas cuyas cuestiones se pueden estudiar mediante el continuo y el discontinuo con una dualidad de métodos que perturban a la Física de hoy por las dificultades que produce y en la Matemática en cambio, reafirman su unidad.

Para Broglie (Louis de, $1892 . .$. , francés) to continuo y lo discontinuo "son como las dos caras de un objeto que no se pueden contemplar a la vez, $y$, sin embargo, hay que tener en cuenta para describirlo completamente" (3), de modo que nuestro conocimiento de los aspectos continuos de la materia aumenta con el conocimiento de sus discontinuidades, y recíprocamente.

En la Matemática, en cambio, las relaciones entre lo continuo y lo discontinuo, lo finito y lo infinito, se pueden enfocar desde dos puntos de vista opuestos: considerar lo continuo como una emanación de lo discontinuo y lo infinito como un enriquecimiento de lo finito o admitir la prioridad de lo continuo y de lo infinito respecto de lo iiscontinuo y de lo finito siendo entonces lo finito una limitación de lo infinito.

La primera posición legitima el paso al límite -operación que tiende el puente entre la Aritmética y el Analisis-y descubre propiedades en lo infinito $\sin$ correspondencia en lo finito. Si consideramos, por ejemplo, un polinomio de variable compleja con un número finito de términos, hay casos en que una serie convergente de infinitos términcs es una función analítica $y$, como ha hecho observar Montel (Paul, 1876...., francés), el paso de un número finito a un número infinito de términos introduce el hecho nuevo de la existencia de un punto singular sin el cual la función analítica se reduciría a una constante (4).

La segunda posición es más filosófica que matemática, ya que lo infinito, en el sentido cartesiano, es anterior a lo finito de igual modo que lo continuo precede a lo discontinuo en la filosofía de Bergson (Henri, 1859-1941, francés).

(3) La Physique nouvelle et les quanta, pág. 242, París, 1937.

(4) Leçons sur les séries de polynomes à une variable complexe, París, 1910.

(Continuará)

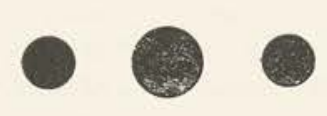

